\title{
The Theory of Natural Movement and its Application to the Simulation of Mobile Ad Hoc Networks (MANET)
}

\author{
Nick Sheep Dalton \\ Open University \\ PO Box 197 Milton Keynes MK7 6BJ \\ n.dalton@open.ac.uk \\ +44(0)1908653153
}

\author{
Ruth Conroy Dalton \\ University College London \\ Gower Street London WC1H0QB \\ r.conory-dalton@ucl.ac.uk \\ +44(0)2076795924
}

\begin{abstract}
The theory of natural movement is fundamental to space syntax: a set of theories and methods developed in the late 1970s that seeks, at a general level, to reveal the mutual effects of complex spatial systems on society and vice versa. In particular, over the years, space syntax analyses have been shown to correlate highly with pedestrian movement and hence are regularly used as a predictive tool, to forecast relative levels of people-flow along streets. Mobile ad hoc networks (MANET) are wireless networks that are selfcreating, having an unfixed and constantly shifting topology. This paper demonstrates how ad hoc networks based on pedestrians carrying mobile wireless devices can be simulated using the theory of natural movement. It suggests that the application of natural movement simulations to mobile ad hoc networks appears to be a useful contribution to the field and that further work should be conducted in this area.
\end{abstract}

\section{Author Keywords}

Mobile ad hoc network (MANET), Vehicular ad hoc networks (VANET), pedestrian movement simulation, agent simulation, space syntax.

\section{Introduction}

Space syntax is a family of theories and analytic techniques that seeks to reveal the intricate interdependencies between space and society [3, 5]. The essence of this dual relationship is that a spatial system (e.g. settlement, neighborhood or complex building) can be considered an artifact of a society and that through the rigorous study of such an artifact/spatial system we can further our understanding of the society that created it.
Conversely, as a society, space is the setting against which all our social interactions, behaviors and encounters take place and these interactions are shaped by the spatial systems that form the backdrop for them. One such behavior that has been shown to be influenced in this way is pedestrian movement. Space syntax methods of analysis are able to accurately predict the relative amounts of pedestrian movement or 'flow' along a linear route. This correlation is accounted for by the theory of natural movement $[4,6$, 7]. This paper seeks to discover whether the theory of natural movement can be usefully applied to the simulation of mobile ad hoc networks. The foundation of the simulation presented in this paper is likely to be unknown to the majority of current researchers in the field and therefore the intended contribution of this paper is the introduction this method of simulation and to explain its novel application to the modeling of mobile ad hoc networks.

\section{The theory of natural movement}

Prior to the theory of natural movement, most models of pedestrian or vehicular movement were based on the concept of land-use 'attractors' embedded in the urban fabric; movement was held as being to and from these attractors, in direct proportion to their relative weights (i.e. essentially a gravity model: some land uses are considered inherently more 'attractive' than others). Numerous, simulated trips are then generated from pairs of origin and destination (O-D) locations and aggregated to produce final pedestrian flow rates. In direct contrast to this model, the theory of natural movement, as proposed by space syntax researchers, is concerned with to-movement from all possible origin-destination pairs within a system. That is to say, every space is considered to be, simultaneously, both an origin and a destination. The through-movement potential of a space is purely the result of the configuration of the urban grid, namely 
the way in which individual components of the urban fabric, main streets, side streets, squares etc. form an interrelated structure. If this structure of streets is represented as a graph it becomes clear that some streets are likely to feature on more topologically shortest routes than others and, in terms of accessibility, are, on average, more accessible than others. This measure of global 'accessibility' is termed 'integration' or through-movement potential in space syntax (similar to 'closeness centrality' in graph theory) and it is this measure that can account for approximately $70 \%-75 \%$ of pedestrian flow rates in some urban environments. The formal reasons for this correlation are set out in Hillier and Iida's 2005 paper [7].

In order to predict the relative flow rates of pedestrians in a complex environment, such as a downtown center or neighborhood, the environment needs to be modeled as a series of discrete lines of potential, unobstructed movement, termed axial lines. The aim of the model is to represent an environment by the least number of such lines of movement. The process of reducing an environment to its key axes of movement can be conducted algorithmically [10] from a base map of building footprints. Figure 1 presents an axial map of the neighborhood of Barnsbury, in London.

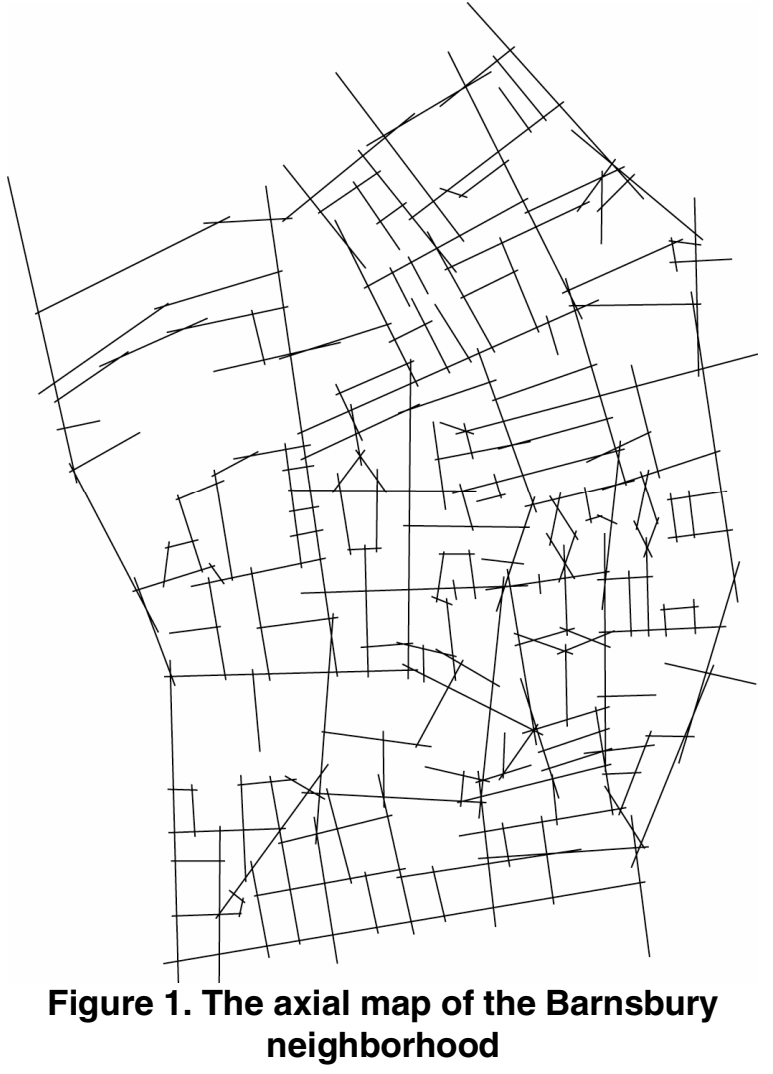

Each axial line is then represented as a node in a graph; wherever any two lines of movement overlap (representing the need for a pedestrian to change direction) the two corresponding nodes in the graph are connected by an edge. This is repeated until all lines and intersections are represented as a meta-graph; the resultant graph is typically non-planar and non-directed (unlike the analogous networks for vehicular movement, which are typically non-planar and directed as they frequently contain one-way streets). Once the graph has been constructed, the integration values are calculated for every line and it is this value that correlates with the numbers of people observed to be traversing that route.

\section{Mobile ad hoc networks}

Mobile ad hoc networks are bottom-up, selforganizing wireless networks. A connection is temporarily made when two devices are in close proximity to one another; once the two devices are out of range the connection is terminated. Hence the topology of an ad hoc network is constantly shifting as new connections are made and existing connections broken. In order to send a message, individual devices need to be able to estimate their own location in relation to the relative locations of proximal devices and their destination. Each handheld device is therefore a multi-channel repeater/transceiver. This type of system can be considered to be bottom-up i.e. it there are no overall pre-determined routes; there is often no centralized service provider and as a network it is fairly robust, providing there is sufficient capacity within the system to permit each device to be within range of one or more other devices (since, if one node drops out of the network, the data packet is able to find another route to its destination).

\section{Problem definition}

In the excellent 2004 paper by Kotz, Newport, Gray, Liu, Yuan and Elliott [1], the authors surveyed an extensive set of simulation models used in the research of mobile ad hoc networks and concluded that the majority of simulations are based on a number of false axioms, namely that:

1. The world is flat

2. A radio's transmission area is circular

3. All radios have equal range

4. If I can hear you, you can hear me (symmetry)

5. If I can hear you at all, I can hear you perfectly

6. Signal strength is a simple function of distance 
(And, indeed, for the sake of simplicity, this paper is guilty of adopting many of the above principles.) However, we would like to suggest that there is a further, seventh, axiom that can be added to this list. The seventh axiom would be that:

\section{People move randomly and are dispersed evenly.}

The reason why this is erroneously considered a truism is that the majority of ad hoc network routing strategy simulations are programmed in unrealistic settings which, in turn, directly affects the behavioral patterns of the agents, resulting in unrealistic patterns of movement [7]. This is particularly problematic as it has a direct and measurable effect on the kinds of ad hoc networks produced, which will be demonstrated in the next section of this paper.

In order to illustrate the difference between these different types of settings ('unrealistic' and 'realistic') two examples have been constructed. The first took place in a fixed-sized 'open' space where the agents were able to moved freely until reaching the boundary: at this point they were forced to change direction. At intervals the agents randomly select a new destination and move towards it. This agent model is similar to many currently used when testing different ad hoc routing algorithms. During this example the agents used a simulated local wireless technology able to create short distance links (relative to the size of the open space). When a link was formed it was plotted on the display (see figure 3 overleaf). This clearly forms a characteristic type of network where nodes have an average connectivity value ranging from 0 to $\mathrm{N}$ where $\mathrm{N}$ is the number of agents present in the open space.

The second example uses a street network covering the same geographic area as the first example. Each agent was provided with a wireless device of the same proximity radius as example 1 and an identical number of agents (one hundred) were released into the urban grid. The difference in this example is that the agents were programmed to move in a manner that has been shown to produce movement patterns analogous to those of real humans in real urban grids. This time, the existence of street networks and the non-uniform distribution of agents on those streets radically altered the configuration of the ad hoc networks created (see figure 4 overleaf). In this case the average degree of a device/node was 3.45 compared to the average degree of a node in the 'open space' experiment, which was 2.33 .

The difference in node connectivities between these two examples can be presented graphically through the use of a frequency distribution, see figure 2 below. The 'open field' connections are dark grey; the 'urban grid' connections are light grey.

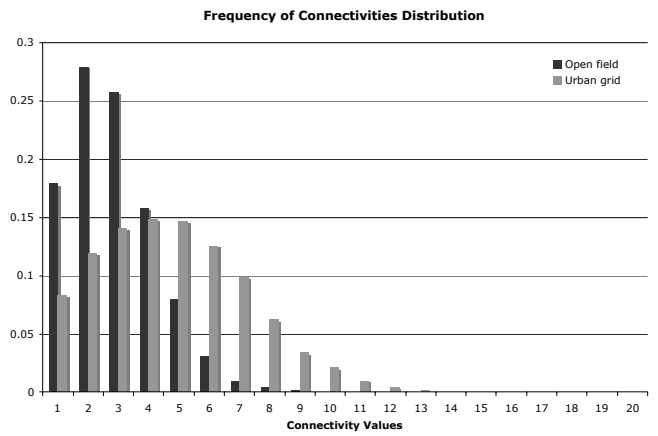

Figure 2. Frequency of device connectivities

It is evident that the urban grid produces denser patterns of connection as it constrains proximity. At the most fundamental level, less geographic area is available for public, pedestrian movement, in the second setting (the urban grid) and therefore, from a purely probabilistic standpoint, higher densities will be produced. However, at a higher level, the street configuration itself will naturally create differential patterns of occupancy (as an emergent phenomenon), whereby some street become, over time, more highly used than others [7].

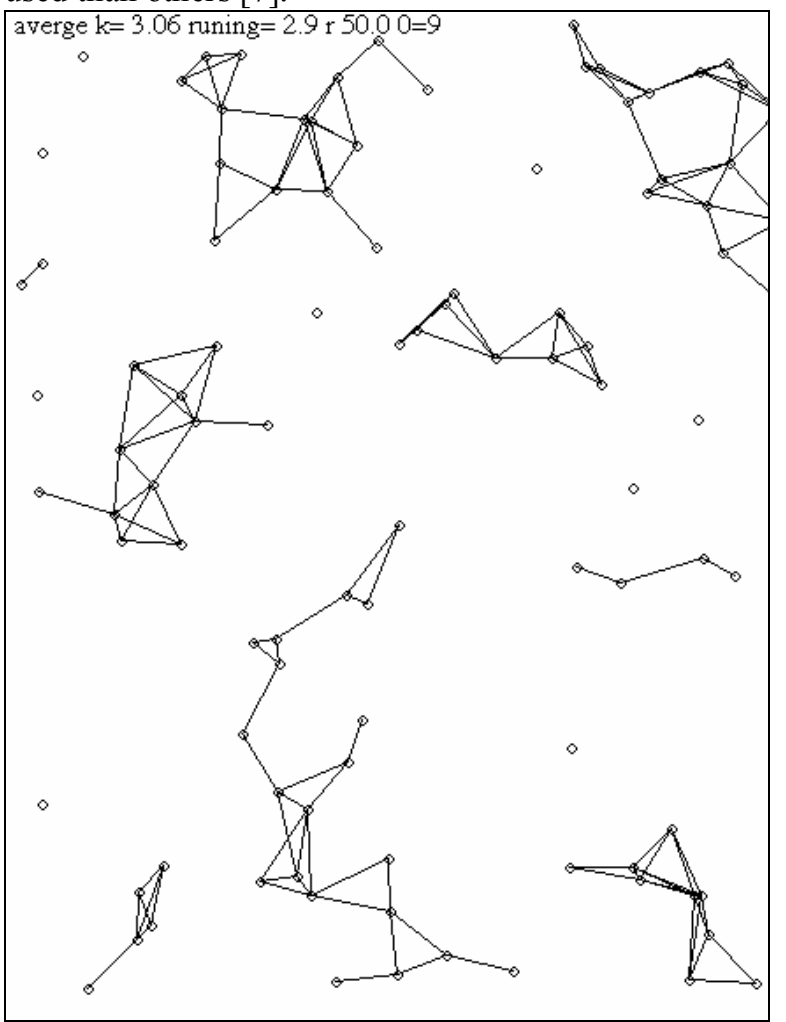

Figure 3. Devices \& ad hoc connections in open space 


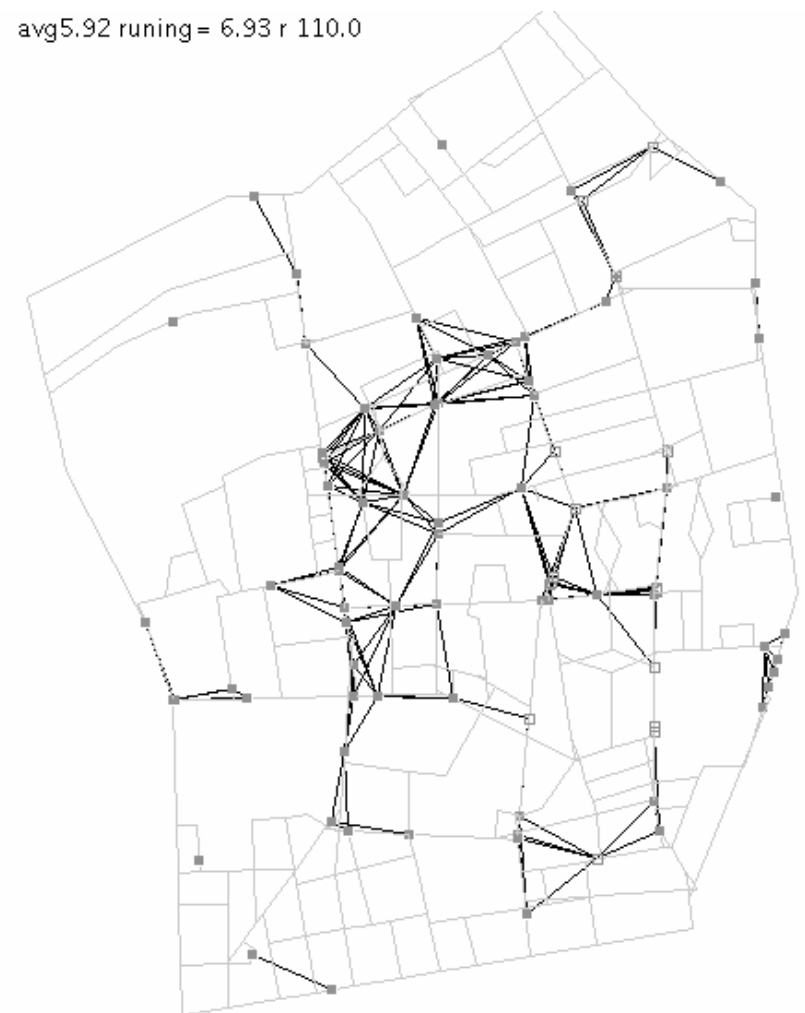

Figure 4. Devices \& ad hoc connections in an urban network

These two examples imply that, when simulating ad hoc network routing strategies, it is imperative to constrain the agents to a realistic setting and to realistic behaviors. This is the aim of the next section of the paper where an ad hoc network simulation based on the theory of natural movement is presented.

\section{The natural movement simulation}

In the simulation of any ad hoc network, possible variables are the total number of mobile devices, the location of devices, the proximity required to transmit between two devices and the rate of data transmission. All of these variables are relatively easy to simulate (given Kotz's axioms) with one exception: in the case of a mobile ad hoc network, what is hard to simulate is the dynamic location of the devices. This is where the theory of natural movement makes a contribution. This simulation tests the efficiency of an ad hoc network in an urban setting, Barnsbury, London (as used already in figures 1 and 4). The first task is to represent this area as an axial map (figure 1) and to create the metagraph that provides the integration values for the model; the agent model is 'seeded' accordingly. Namely, once it has been decided how many devicecarrying people are within the bounds of the neighborhood (and this total is one of the variables of the simulation) they are distributed along the axial lines in direct proportion to the lines' integration values. Those lines that are very integrated within the spatial system will be initially seeded with far more devices than those lines that are quite segregated, which may contain no device-carrying people at all. This is the initial state of the simulation. Upon starting the simulation, each person is assigned a random, physical destination and they select the topologically simplest route through the network (i.e. the fewest number of turns) to reach it. It should be noted that in this simulation, each agent knows its approximate location, insomuch as it knows which axial line it is following as well as its heading. This is broadly analogous to each device, in the real world, determining its own position through the use of GPS or some other type of positioning service [2].

Therefore, every agent/device in the system will have a physical destination. And every packet of data will have a virtual destination. There are effectively two networks overlaid upon each other, each with its own set of destinations. Sometimes, these might be working in harmony and sometimes these two mechanisms might be effectively 'pulling in opposite directions' (an agent walking in one direction, whilst trying to transmit data in the opposite direction).

At the start of the simulation, one person has a data packet that they are attempting to pass along the network to a preset location. Once a message has hopped onto another device (represented as a solid square), there is a time-decay period during which it will have either been passed on to another device or, if it eventually fails to transmit, it is deleted from the device. If a device has a message to pass on it is considered to be 'infectious' (and is represented as a hollow square); if a device has already passed on the message and it has been deleted, it is considered 'immune' to that particular data packet, and any device yet to come in to contact with the message is considered 'non infected'. Throughout the simulation the rates of 'infectious', 'immune' and 'non infected' devices are output as well as the overall ratio between 'infectious' and 'non infected'. The use of these ratios is intended to serve as the simplest baseline test for a network's efficacy. Figure 5 shows one state of the simulation (the ratio of hollow to solid squares).

This basic simulation employs a variant of a 'restricted directional flooding' strategy [8, 9], in which the data packets include their recipient positions. When a device receives a packet, it judges which of the devices are within range and that lie roughly in the direction of its destination and passes on the packet to all of those eligible devices. This process is repeated until the recipient has, hopefully, been reached. In terms of the simulation, each agent knows which other agents are traversing the same axial line and whether they are moving in the same direction or in the 
opposite direction. Depending on the relative direction of a packet's destination, the agent either passes the data onto those moving in the same direction, or those agents passing it in the opposite direction.

In this simulation, the majority of packets arrived at their destinations. The rate of 'infected' devices rose rapidly at the beginning and then began to decrease again; the rate of 'infection' tended towards $90 \%$.

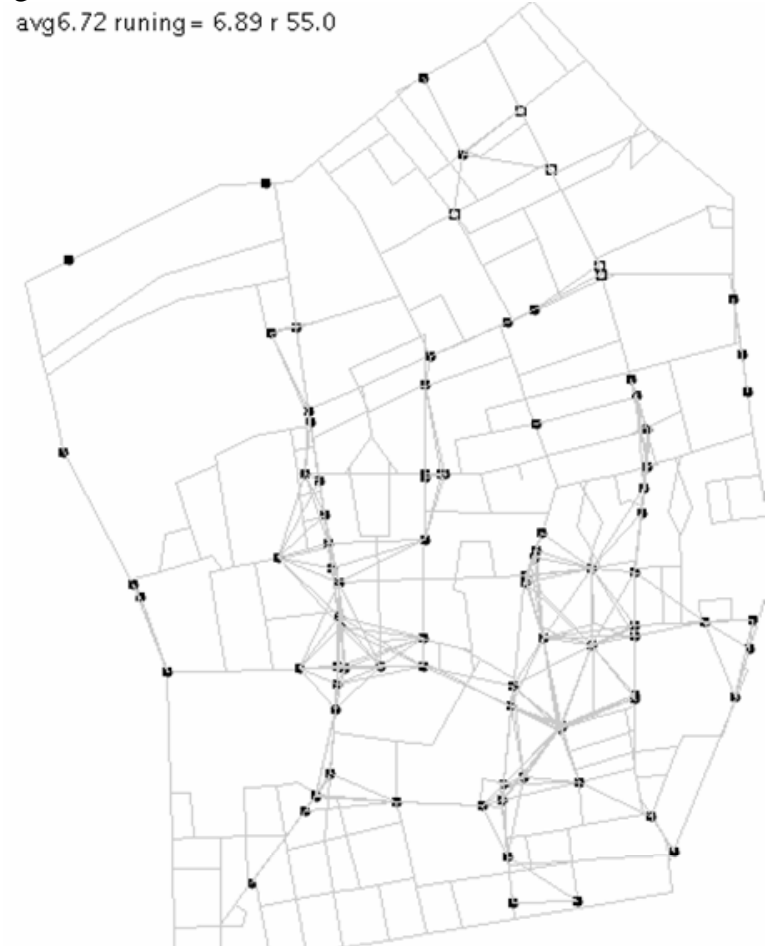

Figure $5.50 \%$ infection rate of agents

\section{Discussion}

This was a tentative 'proof of concept' pilot study in order to determine whether the theory of natural movement could be utilized in such a simulation. Our initial tests have suggested that this could be a useful way of simulating mobile ad hoc networks in complex urban environments and that the way forward should be to try to simulate more sophisticated routing strategies.

In conclusion, we would like to suggest that there are three obvious ways to proceed: increase the accuracy of pedestrian simulations, enhance routing strategies, or both. Since the majority of routing strategies simulations do not account for the fact that people are moving in a natural manner the obvious way forward is to develop both better pedestrian models and better routing strategies and that perhaps the best routing strategies may be those that take advantage of the fact that people do, in fact, move 'naturally'. One such strategy would be to select a 'forward node' on the basis of the integration value of the axial line they are traversing. The theory of natural movement would dictate that there would be a strategic advantage to passing on a data packet to a person walking along a more integrated street than to person on a more segregated one, if only that they are more likely to encounter a greater number of noninfected devices. In future work the authors would like to develop a routing strategy modifying the 'restricted directional flooding' algorithm by prioritizing the most highly integrated devices.

\section{References}

[1] Kotz, D., C. Newport, R. Gray, J. Liu, Y.Yuan and C. Elliott, "Experimental evaluation of wireless simulation assumptions", Dartmouth Computer Science Technical Report TR2004-507, 2004.

[2] Hightower, J. and G. Borriello, "Location systems for ubiquitous computing", Computer, vol. 34, no. 8, 2001, pp. 57-66.

[3] Hillier, B. and J. Hanson, The social logic of space, Cambridge University Press, Cambridge, 1984.

[4] Hillier, B., A. Penn, J. Hanson, T. Grajewski and J. Xu, "Natural movement: or configuration and attraction in urban pedestrian movement", Environment and Planning $B$ : planning and design, 20, 1993, pp. 29-66.

[5] Hillier, B., Space is the machine: a configurational theory of architecture, Cambridge University Press, Cambridge, 1996.

[6] Hillier, B., "Centrality as a process: accounting for attraction inequalities in deformed grids", Urban Design International, 2001, pp. 107-127.

[7] Hillier, B. and Iida, S., "Network and psychological effects in urban movement", In: Cohn, A.G. and Mark, D.M., (eds). Proc. of Spatial Information Theory: International Conference COSIT 2005, Lecture Notes in Computer Science (Vol. 3693). Springer-Verlag, Berlin, 2005, pp. 475-490.

[8] Maihöfer, C., "A survey of geocast routing protocols", IEEE Communications Surveys and Tutorials 6 (2), 2004.

[9] Mauve, M., J. Widmer, and H. Hartenstein, "A survey on position-based routing in mobile ad hoc networks", IEEE Network, vol. 1, no. 6, 2001, pp. 30-39.

[10] Turner, A. and Penn, A. and Hillier, B., "An algorithmic definition of the axial map", Environment and Planning B: Planning and Design, 32 (3), 2005, pp. 425-444 